\title{
Transit Itinerary Calculation on the Web: Based on a Transit User Information System
}

Martin Trépanier, Robert Chapleau, and Bruno Allard Ecole Polytechnique de Montreal

\begin{abstract}
Transit path calculation is not yet widely available on transit authority websites. This is because the calculation of complete transit itineraries requires the integration of underlying components such as Geographic Information Systems (GIS) and operational database and, unlike a simple road application, involves a complex transportation network. In this article, a hybrid algorithm based on heuristics and optimization is presented for the calculation of urban transit itineraries including information on pedestrian access and egress paths, route sequences, schedules, and stops. The use of the Transit User Information System (TUIS) to support the calculations is emphasized. The TUIS uses the Totally Disaggregate Approach (TDA) and Transportation Object-Oriented Modeling (TOOM) in transportation to gather data on territory (for origin and destination specifications and for the pedestrian network) and transit operation (route geometry, schedules). Websites that have been implemented are referenced to demonstrate the applicability of the hybrid algorithm. These websites make use of some special techniques for disseminating user information over the Internet.
\end{abstract}




\section{Introduction}

In general, public transit authority websites fail to offer a complete solution for users who want to obtain transit itineraries between an origin and a destination in a metropolitan area. By January 2000 about 93 percent of public transit websites in the United States still had not integrated an itinerary planning service (Soolman and Radin 2000). Furthermore, the path calculators that do exist on the web rarely provide all the specifications needed by users: origin and destination locations are often limited to a set of locations, and calculations usually do not consider the pedestrian paths built to reach the transit network. Besides, few of these sites provide the schedules associated with the itineraries. Smith (2000) has successfully defined some of the challenges related to this issue. Smith reported that path calculation involves minimizing trip times with respect to spatial, temporal, and system constraints. He maintains that the use of GIS is imperative as a support for the system, and he identified some technologies which could provide the system with flexibility, ease of maintenance, and operability. In the Montreal area, where transit usage is high, research has shown that it is important to provide transit users with the best possible itineraries to facilitate their travel on the network.

In this article, a hybrid algorithm is presented for the calculation of transit itineraries based on a TDA, along with the web architecture and tools needed to set it up on three websites in the Montreal area. With the feature, transit users can now, via the web, have their transit itineraries calculated between any two points in the transit authority's territory. Itineraries are composed of information on pedestrian access and egress paths, bus stops and stations, as well as the set of routes to be taken, full boarding schedule, and transfer, departure, and arrival times. Web users can interactively modify the circumstances of their travel and have the recalculation completed within seconds.

The article presents the research objectives and contributions related to transit path calculation on the web. Then, after providing a conceptual background, it describes three methodological developments associated with the TUIS: the TDA, TOOM, and Object-Oriented GIS (OO-GIS). This is followed by the development of the hybrid algorithm, which is divided into four major phases: (1) origin, destination, and circumstance specification; (2) access and egress calculation; (3) path calculation; and (4) schedule integration. Finally, three examples of tested website environments are reviewed. To facilitate the discussion, relevant elements of a literature review are presented along with the topics. 


\section{Research Objectives}

In the mid-1990s, the Montreal Urban Community Transit Commission (MUCTC), a 65-station subway and 1,500-bus operator, expressed the need to computerize the activities of its 20 -agent phone information center to increase the productivity of its phone agents and to broaden the range of informational tools available to its users. An initial research project led to the implementation in 1995 of the software MADPREP (Module d'Acquisition de Données pour la Préparation de la Réponse Émise par le Préposé, data entry module for the preparation of agent responses) (Chapleau et al. 1997). The technology has since been upgraded and installed in two other transit information centers in the Montreal area.

Given the continuing growth and popularity of the Internet, another research project was initiated to set up a website for the MUCTC. The first objective was to provide users with static information on transit services; the second objective was more challenging: to give users web tools for calculating transit itineraries. Typically, the information associated with transit paths would include:

- multimodal (transit and pedestrian) paths from point A to point B located within the territory;

- stops, routes, transfer points, and schedules related to the itineraries; and

- estimated walking, waiting, transfer, and travel times.

Figure 1 shows a graphical representation of the transit path information.

Since no commercial software or actual model could fulfill MUCTC needs, a new method was developed based on the TDA, TOOM, and OO-GIS.

\section{Background}

This section examines the three methodological developments of the TUIS.

\section{TDA}

The TDA was developed in the 1980s in Greater Montreal for the validation, processing, and modeling of large, origin-destination surveys among householders, conducted particularly with a view to gather data on transit usage. Typically, in 1998 , a phone survey would involve more than 70,000 households ( $5 \%$ overall sampling). Planners, however, were not satisfied with the way in which such a quantity of data was being modeled with an aggregate approach, even with a 1,500-zone system (Chapleau 1986). A new method had to be developed to store and process 
Figure 1 . The totally disaggregate trip

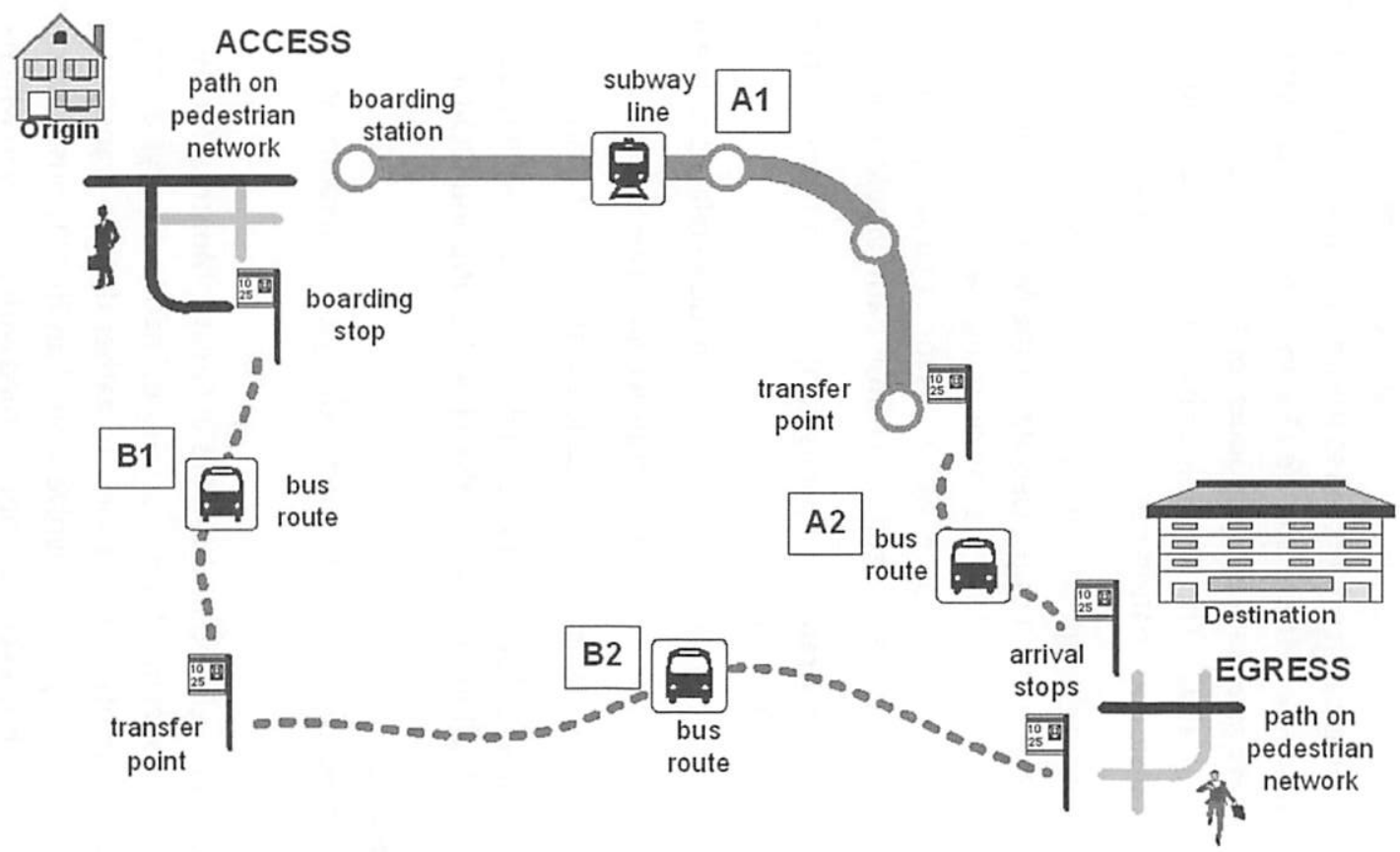


data on households, individuals, and trips. The TDA can be quickly defined by the following two essential elements:

1. The focus throughout the transportation analysis is the individual trip. All trip characteristics (time, purpose, modes, itinerary) are maintained in association with the individual and household concerned.

2. $X-Y$ coordinates, monuments, and statements about places are used to form the basic, spatially referenced system for the origin, destination, and residential and intermodal junction locations of each trip and other spacerelated objects in the system.

In terms of data completeness, the TDA does not use an origin-destination matrix, as this would aggregate and dissolve information. It maintains origin-destination files instead, which contain survey information on trips, individuals, and households (see Figure 1). The use of the best-defined information improves the level of resolution of the system without preventing any possible aggregation.

Over the years, many applications have been developed to enrich the data in these databases by adding new attributes (e.g., status derivation from a daily activity or from car ownership) (Chapleau et al. 1998). One of these applications is the trip calculator, which was first used to validate the transit trips described in surveys. Once calibrated with survey data, the calculator is used to simulate trips on the transit network. It has been used in several models and studies on trip generators, financing (Chapleau 1995), modal split (Chapleau and Trépanier 1997), etc. The itinerary calculation process presented in the section on OO-GIS is based on this methodology.

\section{TOOM}

TOOM is derived from object-oriented schemes used in traditional approaches like those of Booch, Coad, Colbert, Yourdon, and others (Trépanier and Chapleau 2001a). Viewed in this way, the object is a unique entity with its own properties and methods. Its properties (attributes) describe the object's state. Its methods constitute the behavior of the object: its actions and its services to other objects.

The transportation object is a special component intended for modeling, observing, planning, and analyzing the transportation system. For this purpose, its state is variable in time and space and possesses special properties and methods. For example, a Roadlink object has the commonly used road properties (length, name, number) but can also have time-varying properties (e.g., pavement condition). 
Figure 2. Simplified object model of the transit user itinerary.

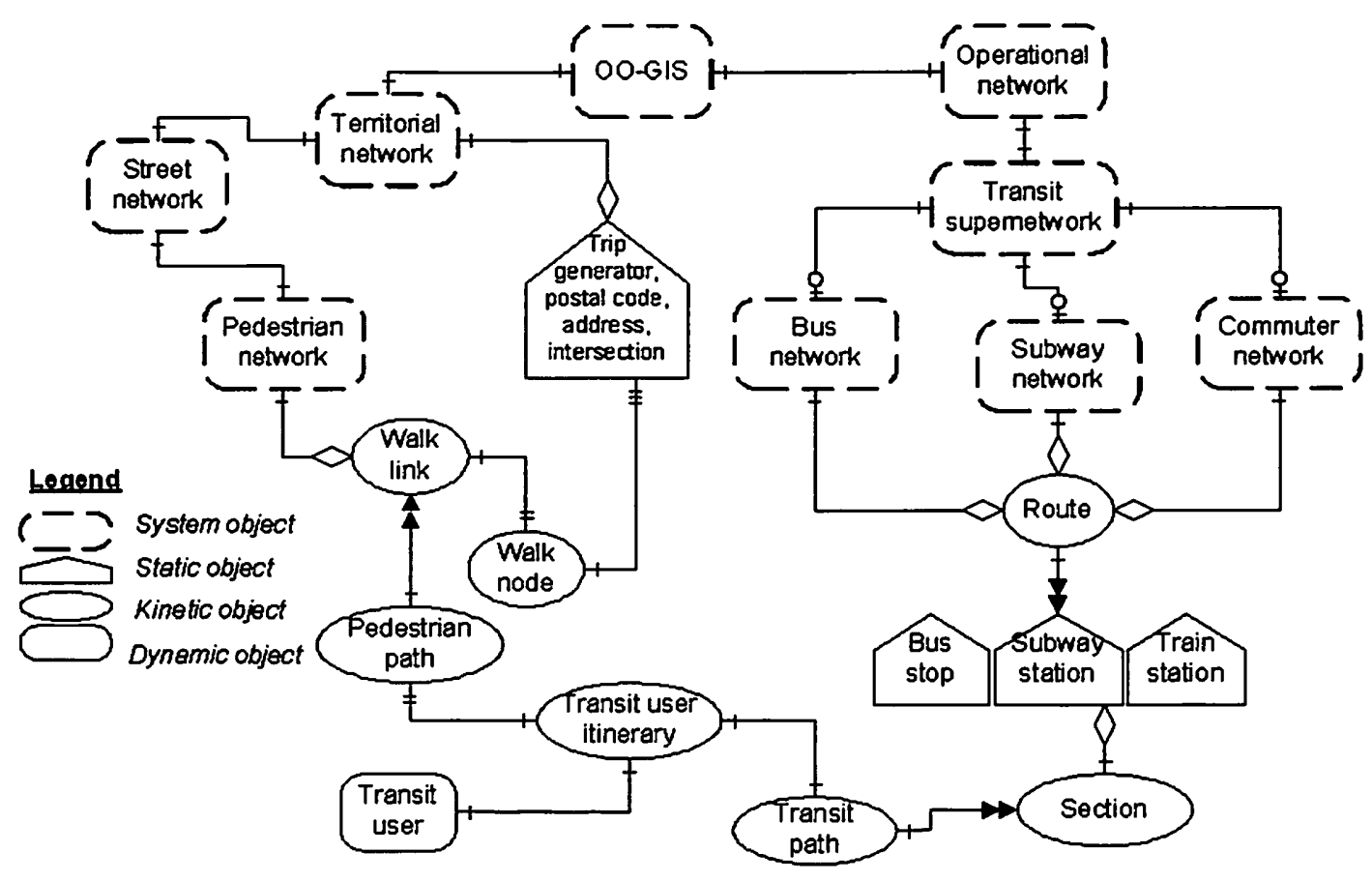


There are four metaclasses of transportation objects involved in dynamic and spacerelated relations, depending on their specific role:

- Immobile (static) objects have a fixed location in time and space. Their role is to describe the territory and serve as transportation movement beacons. Some examples are: TripGenerator, PostalCode, CensusTract, and Zone objects.

- Dynamic objects are the transportation actors. They decide on, and contribute to, their movements. They include a group of individuals (Household objects, Person objects), moving objects (Bus, Car), or even objects moved (Goods).

- Kinetic objects are objects that describe movement. Some examples are: Trip, TransitLink (simple kinetics), Path, and TransitRoute (compound kinetics).

- System objects are groups of embedded objects with their set of relations. They can be operational (TransitNetwork, RoadNetwork), informational (Survey, Census), or globally comprehensive (UrbanSystem).

A transportation method is an "intelligent" sequence of procedures used to manipulate and transform one or more transportation objects. It blends models with information, creating "infomodels" to be reapplied to similar objects. TOOM is not primarily aimed at software design or database structure, and it is not a database issue. First and foremost, it is a "way of thinking" about the role and specific use of every piece of information in the system. With adequate diagrams, objects can be rapidly identified, along with their properties and methods involved in the analysis. The software implementation could easily integrate these underlying concepts, but not all software languages are adapted to this methodology.

\section{0-GIS}

The use of TOOM is particularly important for the implementation of GIS within a transit authority's territory (Trépanier and Chapleau 2001b). This kind of GIS is unfamiliar, since it integrates a transit network, a road network, and territorial data simultaneously (Peng, Groff, and Dueker 1998). In Figure 2, the object model for a typical path calculation is represented schematically. The transit supernetwork describes the geometry and the chronometry (time-related aspects) of transit operation. For a given authority, it includes all modes; in the Montreal case, the bus, subway, and commuter train networks are integrated. The territorial network describes land use, the street network, and the sidewalk network (pedestrian). This 
Figure 3. Transit User Information System architecture.

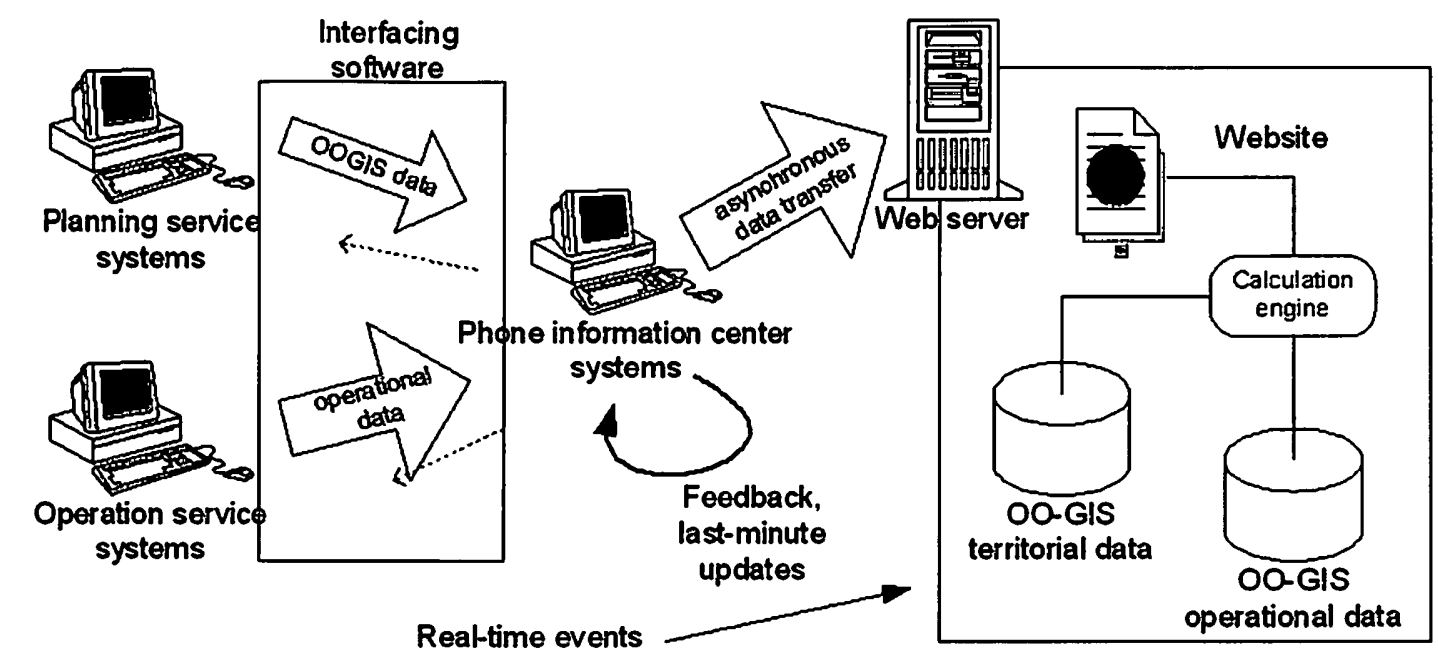


third object is mandatory to provide adequate information to the transit user, since access to transit services is a pedestrian path and must be considered. The system also integrates some "classic" GIS information [e.g., addresses, intersections, postal codes, trip generators (monuments)]. These objects are used for origin and destination specifications. At the bottom of the object model, the transit user is provided with two elements of information: the user path on the pedestrian network, and the itinerary for arriving at the transit network.

\section{TUIS}

Some effort has been made to describe the elements that could be used to supply information to a transit website. Ventura County was among the first to provide transit service information on the web (Gherardi et al. 1997). Lee, Baumgartner, and Tschango (1999) presented a web-based bus information system (WBI) implementation with data stored in a Microsoft Access database. Peng and Huang (2000) also used Access to store route and transit networks, bus stops, and time-point data, and their database is linked to GIS and network servers (Peng and Beimborn 1998).

\section{Architecture}

In terms of implementations of our websites, the following architecture has proven to be an adequate means of providing transit trip itinerary calculation on demand via an Internet interface (about 80,000 calculations in the year 2000). The whole system is called the TUIS for the web and is represented in Figure 3.

As shown previously in Figure 2, OO-GIS integrates two groups of data: operational and territorial. Transit agencies usually already have systems in place to manage data related to vehicle operations (e.g., driver schedules, vehicle dispatch times, runs, schedules). At the MUCTC, many software programs are used for these tasks. Interface software has been developed through this project to gather, integrate, and validate data from these systems. In contrast, territorial data are commonly used in the planning department and stored in GIS architecture. Another program processes these data to adapt them to the OO-GIS model.

Data are transmitted through the phone information center service. Typically, a copy of the data is installed there shortly before its implementation. This copy is installed in the phone information center so that the agents, who probably have the best knowledge of the operational and territorial networks in the organization, can then test the calculator to detect problems and initiate last-minute changes, such as new streets (new streets may not immediately be included on official city 
Figure 4. Elements of a TUIS for thw web.
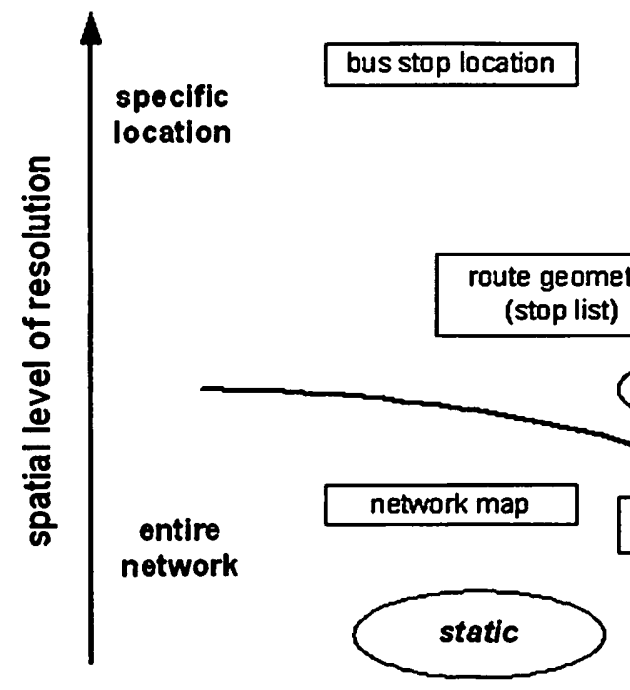

long term (months, years)
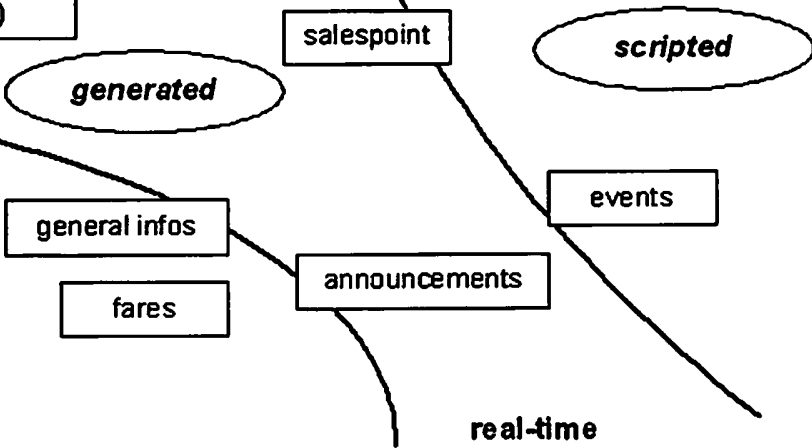

(thours, days) 
maps) or modified schedules (these small changes may not be reported to the corporate information systems department).

The architecture of the web server, which is quite simple, is shown in Figure 3. The PC based server physically integrates all the components: database (operational and territorial data in a Visual FoxPro application), web server (Internet Information Server), and calculation engine (Windows DLL dynamic library). The major part of the data are asynchronously transferred to the web server when needed. Events and other real-time data are transferred through TCP/IP communications between application servers.

\section{Spatial and Temporal Level of Resolution}

A common question related to user information concerns the level of spatial and temporal resolution needed to satisfy the user. Should it be a simple on-demand route schedule or a stop schedule? Should the transit operator provide a full transit path between two points in its territory? In the Montreal area, authorities had accustomed the user to receiving precise and detailed information, including, for example, the full pedestrian network for reaching bus stops. This information had historically been provided by phone by experienced agents, with no computerized tools, only paper-based documents like maps and schedules.

Spatial and temporal levels of resolution are two dimensions to consider when designing a TUIS for the web (see Figure 4). As the spatial level of resolution increases, the spatial definition of transit information becomes more precise, and the number of web elements (e.g., bus stop location pages) increases. The same logic is applied to the temporal level of resolution. These two dimensions have an impact on the technical structure of the website, so that several web technologies must be used to render the information with efficiency and without overloading the web server. Individualized schedules and calculated itineraries constitute on-demand, real-time information that must be provided by the web server via scripted pages (e.g., Active Server Pages, Common Gateway Interface). The path calculation process described in this article is illustrated in the upper-right-hand part of the chart. In contrast, general information on the transit authority is usually provided via static HTML pages to improve the server's performance (Kothari and Claypool 2001). The central zone includes generated pages, containing a large number of data, like route geometry, bus stop lists, planned schedules, and point-of-sale lists. To avoid overloading the web server, this information is not scripted, but rather generated by a database software program used to make the several thousand HTML pages. 
Figure 5. The itinerary calculation process.

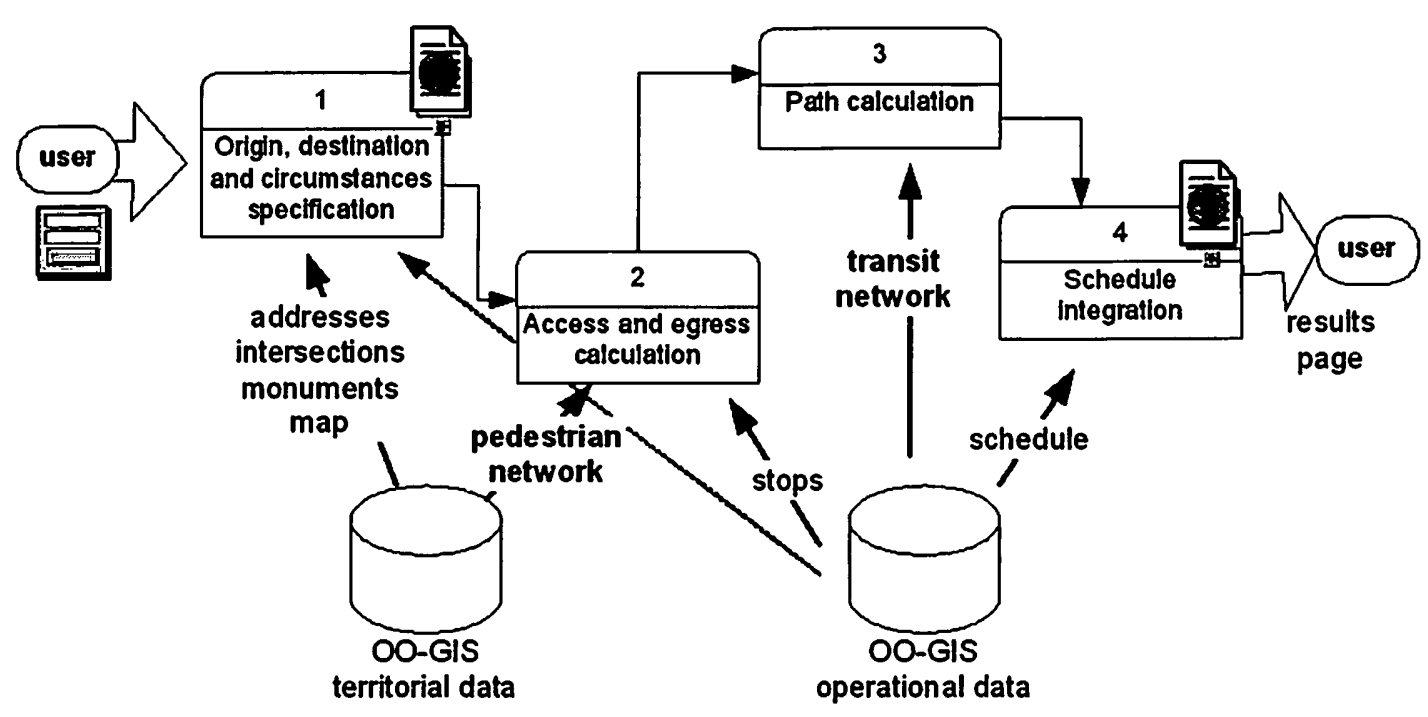




\section{Itinerary Calculation Process}

Although many transit agencies have implemented websites in recent years, few provide adequate transit itinerary service via this medium. Peng and Huang (2000) have indicated that a network analysis model is "the key component" to providing trip itinerary planning and reported that most algorithms and programs are designed for highway calculation use. In fact, transit path calculation is much more complex than road network itinerary calculation because it involves different transportation modes, time-dependent levels of service, and the presence of transfer points, terminals, stops, and stations.

The transit path calculation methodology presented in this article consists of four steps (Figure 5): (1) origin, destination, and circumstance specification; (2) access and egress calculation; (3) path calculation; and (4) schedule integration.

\section{Origin, Destination, and Circumstance Specification}

The calculation process begins with a choice of origin and destination. The user then has to define the circumstances of the itinerary. To determine location, an HTML form is used to enter an address (civic number + street name), intersection (two street names), monument (interactive search or browsing of a list), or station or bus stop number. The web server, with the help of the underlying territorial OO-GIS, decodes the form and stores the locations as user preferences. An interactive, clickable map is also provided as an alternative way to define origin and destination, supported by a bitmap-based web GIS. Any reachable location in the agency's territory (not simply a restricted list) can be specified as an origin or a destination. Under "Circumstances," the user enters the travel day and time and selects options related to subway, train, and pedestrian usage. The user can choose not to use the subway, for example, and ask for the shortest access distance, thereby increasing the number of transfers but minimizing walking distance.

\section{Access and Egress Calculation}

In the next step, a pedestrian path calculation is used to gather a set of bus stops (or train and subway stations) around the origin and the destination. To identify this set, a shortest-path tree is calculated on the pedestrian network from the origin location. The same task is performed for the destination. At least four stops are gathered at each end, since the nearest stop would not necessarily provide the best transit path. (This number is parametrized according to the sensitivity of the model.) The use of the pedestrian network (sidewalks, pedestrian paths, underground paths, and pedestrian overpass bridges) is very important to avoid confusing users. The 
pedestrian network must consider geographical obstacles such as train tracks, rivers, and freeways.

\section{Path Calculation}

The set of stops located at the origin and destination is then used to calculate several transit paths. Peng and Huang (2000) suggest that some elements of the paths should be precalculated to speed up the calculation. In our applications, it would be unrealistic to prestore the information because the number of stops (more than 16,000), number of routes (more than 500), and the temporal dynamic aspects of the transit network would generate millions of possible paths. Another reason not to prestore the information is that the calculation is performed within a fraction of a second on a regular PC. The calculator uses a shortest-path algorithm (adapted from Dijkstra) and heuristics (to rearrange the network) to determine a set of feasible itineraries between the stops and relies on the transit network definition of the MADITUC software in the TDA (Chapleau 1986). The shortest paths are calculated such that the total generalized travel time $\left(t_{\text {total }}\right)$ obtained from this impedance function is minimized:

$t_{\text {total }}=w^{m} t_{o}^{m}+\sum_{1}^{n}\left(w^{a} t_{n}^{a}+t_{n}^{p}\right)+w^{m} t_{d}^{m}$

where:

$t_{o}^{m} \quad$ is the access time (walk at origin).

$t_{d}^{m} \quad$ represents the egress time (walk at destination).

$t_{n}^{p} \quad$ is the travel time on-board route $\mathrm{n}$ vehicle.

These times are estimated from an average walking speed (about $4 \mathrm{~km} / \mathrm{h}$ ) and highway speeds derived from schedules. Weighting factors $w^{m}$ (walk) and $w^{a}$ (waiting) are applied to balance the impedance. The waiting time for route $\mathrm{n}, t_{n}^{a}$, takes into account several components:

$t_{n}^{a}=\min \left(\max \left(t_{\min }^{a}, w_{n}^{x} h_{n}^{i}\right), t_{\max }^{a}\right)+t^{t} w_{n}^{f}+w_{n}^{n-1}$ 
where:

$t_{\min }^{a} \quad$ is the minimum waiting time (boarding time at a minimum).

$t_{\max }^{a} \quad$ represents the maximum waiting time (over that time, the user will adjust this time in accordance with the schedule, especially for large headways).

$w_{n}^{x} \quad$ is the weighting factor for the waiting time related to headway $h_{n}^{t}$. This waiting factor depends on the location on the route ( 0.5 near the beginning). The second term is the transfer time ( 0 for the first route) and implies $t^{\prime}$ the base transfer time and $w_{n}^{f}$ the weighting factor related to the route mode (could be higher for a commuter train, for example).

$w_{n}^{n-1} \quad$ is an additional penalty for mode transfer between route $n-1$ and route $n$. This term of the impedance function can be used to consider rates dues to mode change.

This impedance function is calibrated with the help of household survey data. More than 35,000 origin-destination pairs were extracted from the 1998 survey and simulated with the algorithm to reproduce the transit statements in the survey (sequences of routes taken). Weighting factors also serve the user on-line on the web. For example, if the walk is to be minimal, its weighting factor () in the impedance function will be increased so that the calculator will try to minimize the walk-time terms.

\section{Schedule Integration ("Trimming")}

Once the calculator has produced feasible itineraries, the operational OO-GIS is used, via a database management software program (DBMS), to add detailed schedule information. In some cases, the calculator provides more than 50 itineraries ( 4 stops at origin $X, 4$ stops at destination $Y, 3$ or more feasible paths), and so a choice must be made to retain the two or three "best" paths. This is done by recalculating the total travel time for each itinerary using a schedule, with the following formula: where:

$h_{0}^{u} \quad$ is the starting time specified by user.

$h_{n}^{o} \quad$ is the scheduled departure time for route $n$.

$h_{n}^{d} \quad$ represents the scheduled arrival time.

$t_{o}^{m}, t_{d}^{m}$ is the walking time. 
The total travel time is then provided in minutes and is the difference between the itinerary's overall arrival and starting times. This part of the process is called schedule "trimming."

\section{Implementations}

Experiments on the path calculation methodology were first conducted on a large transit agency website and were extended to a medium-sized transit agency website and a suburban transit agency. In 2001, about 462,000 path calculations were performed on the MUCTC website ( 80,000 in 2000).

Transit users and web surfers go onto the MUCTC website (http://www.stm.info) to calculate transit paths with the "Tous Azimuts" (all azimuths) function (Figure 6). The calculation results (one to three paths) are provided in a table format, containing: starting time from the origin location and walking time to the access stop, schedule at each stop (access, egress, and transfer), stop and route identification, pictograms for transit mode (bus, train, subway), phone numbers (for phone scheduling system), arrival time at destination location, and total travel time.

At the City of Laval Transportation Agency, a suburban transit network north of Montreal, the results are displayed in text form. The site is located at: http:// www.stl.laval.qc.ca. (Text is provided in French only). The phrasing used by the web server software contains the same information as the MUCTC results table. In this case, the pedestrian and transit networks are larger, since they integrate both the

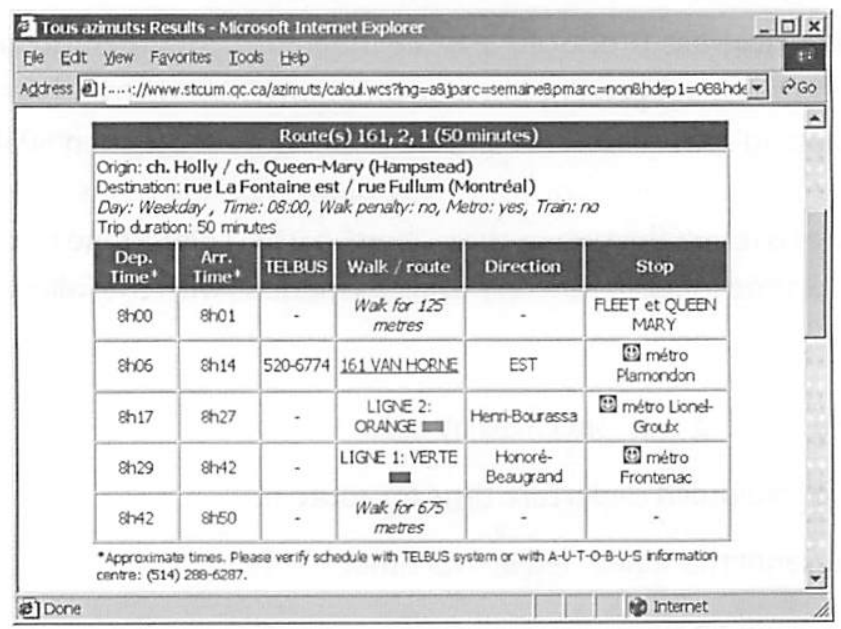

Figure 6. Calculation results on the MUCTC website. 
Montreal and Laval territories. Users can also store up to 10 origin and destination locations via cookie files. Precise schedules are provided for the Laval network only, and the user is invited to browse the Montreal schedule through hyperlinks to the MUCTC website.

The third implementation of the calculator provides transit access information (set of bus stops) around a location within the Greater Montreal area $\left(5,000 \mathrm{~km}^{2}\right)$. It is available on the Metropolitan Transportation Agency website ( $h t t p: / /$ www.amt.qc.ca, also in French only). The agency is responsible for the commuter bus and train services provided by about 20 small operators. A regional transit path calculator is currently under development and is awaiting the completion of a regional TUIS to gather and structure available data.

\section{Conclusions}

It is possible for transit users to use the web to calculate transit paths for their daily trips. In Montreal, where there is a large and multimodal network in place and where schedules are revised every two months, such a function has been very helpful.

This article has explored a transit itinerary calculation methodology based on a hybrid heuristic algorithm that relies on a TUIS. The TDA, TOOM, and OO-GIS are the three elements developed to support the algorithm and its embedding in websites. The article also shows that a powerful means of integrating information at each stage of the process is key to obtaining a viable algorithm for providing suitable itineraries for users.

Some challenges remain to be addressed, and these will be explored in the coming years. First, there is a possibility that path calculation will be used in some planning processes such as route synchronization or delay studies. Second, the most powerful integration of real-time events and on-board GPS data will be examined. Finally, a more sophisticated version of the algorithm for calculating hybrid road-transit paths for the use of park-and-ride facilities for commuters is under consideration. 


\section{Acknowledgments}

The authors wish to express their gratitude to this project's partners: the Montreal Urban Community Transit Commission (Société de transport de la Communauté Urbaine de Montréa), City of Laval Transportation Commission (Société de transport de la Ville de Lava), and Metropolitan Transportation Agency (Agence métropolitaine de transport). They also thank the Natural Sciences and Engineering Research Council of Canada. 


\section{References}

Chapleau, R. 1986. Transit network analysis and evaluation with a Totally Disaggregate Approach. Vancouver: World Conference on Transportation Research Proceedings.

Chapleau, R. 1995. Measuring impacts of transit financing policy in geopolitical context: Montreal case. Transportation Research Record 1496: 52-58.

Chapleau, R., and M. Trépanier. 1997. Transportation Object-Oriented Modeling: An Extension of the Totally Disaggregate Approach. Austin, TX: International Association of Travel Behavior Research.

Chapleau, R., B. Allard, and M. Trépanier. 1997. Transit path calculation supported by a special GIS-transit information system. Transportation Research Record 1521: 98-111.

Chapleau, R., M. Trépanier, and B. Allard. 1998. Practical implementations of object-oriented GIS-T. Anvers, Belgium: World Conference on Transportation Research.

Gherardi, G., S. DeGeorge, and A. Aune. 1997. Point-to-point transit routing: On the World Wide Web. American Public Transit Association Bus Operations, Technology and Management Conference, Miami.

Kothari, B., and M. Claypool. 2001. Dynamic web pages: Performance impact on web servers. Internet Research: Electronic Networking Applications and Policy 11 (1): 18-25.

Lee, J. S., J. A. Baumgartner, and J. K. Tschango. 1999. A web-based bus information system. ESRI User Conference Proceedings 494.

Peng, Z.-R., and E. A. Beimborn. 1998. Internet GIS and its applications in transportation. Transportation Research News 195 (March-April): 22-26.

Peng, Z.-R., J. N. Groff, and K. J. Dueker. 1998. An enterprise GIS database design for agency-wide transit applications. URISA Journal 10 (2).

Peng, Z.-R., and R. Huang .2000. Design and development of interactive trip planning for web-based transit information system. Transportation Research Part C: Emerging Technologies (8): 409-425.

Smith, B. L. 2000. Using Geographic Information Systems and the World Wide Web for interactive transit-trip itinerary planning. Journal of Public Transportation 3 (2). 


\section{About the Authors}

MARTIN TRÉPANIER (mtrepanier@polymtl.ca), P. Eng., Ph.D., is an assistant professor of industrial engineering at the Ecole Polytechnique de Montreal. His research is mainly related to logistics, information systems, object-oriented modeling, GIS development, and Internet applications. He worked along with the MADITUC group in this research project.

Robert Chapleau (rchapleau@polymtl.ca), P. Eng., Ph.D., is a professor of civil engineering (transportation planning) and founder-director of the MADITUC group, Civil Engineering Department, Ecole Polytechnique de Montreal. He developed the Totally Disaggregate Approach in transportation and is participating in several research projects in the Montreal area.

BRUno AllaRD (ballard@polymtl.ca), P. Eng., M.Sc.A., is a research associate in the MADITUC group, Civil Engineering Department, Ecole Polytechnique de Montreal. He is an experienced system developer and manager, with applications in origindestination surveys, transit operation, and GIS-T-based websites. 